\title{
Searching for the Missing Baryons in the Warm-hot Intergalactic Medium
}

\author{
Philipp Richter*t \\ IAEF Bonn, Germany \\ E-mail: prichtereastro.uni-bonn.de
}

\section{Blair D. Savage}

Department of Astronomy, University of Wisconsin-Madison, USA

\section{Todd M. Tripp}

Department of Astronomy, University of Massachusetts, USA

\section{Kenneth R. Sembach}

Space Telescope Science Institute, USA

\begin{abstract}
We discuss physical properties and the baryonic content of the Warm-hot Intergalactic Medium (WHIM) at low redshifts. Cosmological simulations predict that the WHIM contains a large fraction of the baryons at $z=0$ in the form of highly-ionized gas at temperatures between $10^{5}$ and $10^{7} \mathrm{~K}$. Using high-resolution ultraviolet spectra obtained with the Space Telescope Imaging Spectrograph (STIS) and the Far Ultraviolet Spectroscopic Explorer (FUSE) we have studied the WHIM at low redshifts by searching for intervening O VI and thermally broadened Lyman $\alpha$ (BL) absorption toward a number of quasars and active galactic nuclei (AGNs). Our measurements imply cosmological mass densities of $\Omega_{b}(\mathrm{O} \mathrm{VI}) \approx 0.0027 h_{75}^{-1}$ and $\Omega_{b}(\mathrm{BL}) \approx 0.0058 h_{75}^{-1}$. Our results suggest that the WHIM at low $z$ contains more baryonic mass than stars and gas in galaxies.
\end{abstract}

Baryons in Dark Matter Halos

5-9 October 2004

Novigrad, Croatia

\footnotetext{
*Speaker.

${ }^{\dagger}$ DFG Emmy-Noether Fellow
} 


\section{Introduction}

Weakly and highly ionized intergalactic gas most likely makes up for most of the baryonic matter in the local Universe. While the diffuse photoionized intergalactic medium (IGM) that gives rise to the Lyman $\alpha$ forest is expected to account for $\sim 30$ percent of the baryons today (Penton, Stocke, \& Shull 2004 [1]), the highly-ionized Warm-Hot Intergalactic Medium (WHIM) at temperatures $T \sim 10^{5}-10^{7} \mathrm{~K}$ possibly contributes at a comparable level to the cosmological mass density of the baryons at $z=0$ (e.g., Davé et al.2001 [2]). Gas and stars in and around galaxies and galaxy clusters make up the rest (Fukugita 2003 [3]). The total cosmological mass density of the baryons, $\Omega_{b}$, has been restricted to a value of $\Omega_{b} \approx 0.04$, e.g., by measuring the deuterium-to-hydrogen ratio in high-redshift absorption line systems (Burles \& Tytler 1998 [4]) and by analyzing the small-scale anisotropy of the cosmic-microwave background (Spergel et al. 2003 [5]). However, the contribution from the WHIM to $\Omega_{b}$ has been estimated mainly from numerical simulations of the structure formation in the Universe rather than from observational results (e.g., Cen \& Ostriker 1999 [6]; Davé et al. 2001 [2]). So far, only a few percent of the baryonic matter residing in the WHIM at $z=0$ actually has been detected. To test the cosmological simulations and to learn about the distribution of the baryons in the local Universe it is of crucial importance to look for possibilities to pinpoint the baryon budget in the WHIM by direct observations.

\section{O VI Absorbers}

Five-times ionized oxygen ( $\mathrm{O}$ VI) currently is the most important high ion to trace the WHIM at temperatures of $T \sim 3 \times 10^{5} \mathrm{~K}$ in the FUV regime. Oxygen is a relatively abundant element and the two available O VI transitions (located at 1031.9 and $1037.6 \AA$ ) have large oscillator strengths. A number of detections of intervening WHIM O VI absorbers at $z<0.5$ have been reported in the literature (see Sembach et al. 2004 [7] and references therein) based on observations with HST/STIS and FUSE. These measurements imply a number density of $\mathrm{O}$ VI absorbers per unit redshift of $d N_{\mathrm{OVI}} / d z \approx 14$ for equivalent widths $W_{\lambda} \geq 50 \mathrm{~m} \AA$, as derived from the analysis of six lines of sight (Sembach et al. 2004 [7]). Assuming that 20 percent or less of the oxygen is present in the form of O VI $\left(f_{\mathrm{OVI}} \leq 0.2\right)$ and further assuming a mean oxygen abundance of 0.1 solar, the measured number density corresponds to a cosmologial mass density of $\Omega_{b}(\mathrm{O} v \mathrm{~V}) \approx 0.0027 h_{75}^{-1}$. For the interpretation of this value it has to be noted that O VI absorption mainly traces gas with temperatures around $3 \times 10^{5} \mathrm{~K}$, but not the million-degree gas phase which probably contains the majority of the baryons in the WHIM. Very recently, Savage et al. (2004 [8]) have reported the detection of Ne VIII in an absorption system at $z \approx 0.2$ in the direction of the quasar HE $0226-4110$. Ne VIII traces gas at $T \sim 7 \times 10^{5} \mathrm{~K}$ and thus is possibly suited to complement the $\mathrm{O}$ VI measurements of the WHIM in a higher temperature regime. However, as the cosmic abundance of neon is relatively low, it is not expected that Ne VIII is a particularly sensitive tracer of the WHIM. This is supported by the non-detections of intervening Ne VIII in other high S/N STIS data (Richter et al. 2004 [9]). 


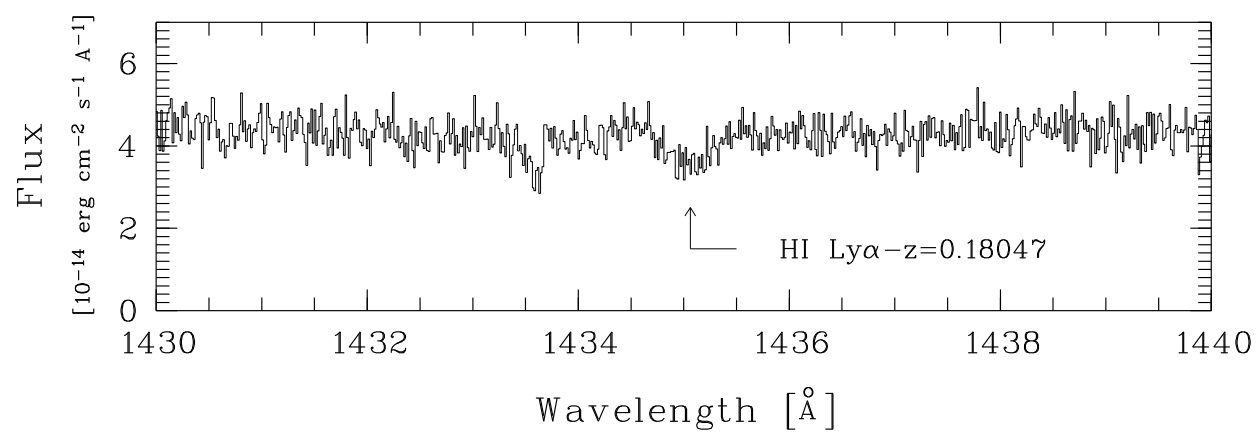

Figure 1: Broad Ly $\alpha$ absorber in the STIS spectrum of H $1821+643$ at $z=0.18047$. This absorber has a $b$ value of $56 \mathrm{~km} \mathrm{~s}^{-1}$ and an $\mathrm{H}$ I column density of $\log N=13.21$. For pure thermal broadening and assuming CIE, these numbers imply a gas temperature of $\sim 2 \times 10^{5} \mathrm{~K}$ and a total hydrogen column density (neutral + ionized) of $\log N=18.61$.

\section{Broad Lyman $\alpha$ Absorbers}

Next to high-ion absorption from oxygen and other metals, recent observations with STIS (Richter et al. 2004 [9]; Sembach et al. 2004 [7]) suggest that WHIM filaments can be detected in Ly $\alpha$ absorption of neutral hydrogen. Although the vast majority of the hydrogen in the WHIM is ionized, a tiny fraction $\left(<10^{-5}\right.$, typically) of neutral hydrogen should be present if the gas is in collisional ionization equilibrium (CIE; see Sutherland \& Dopita 1993 [10]). Depending on the total gas column density of a WHIM absorber and its temperature, weak H I Ly $\alpha$ absorption at column densites $12.5 \leq \log N(\mathrm{H} \mathrm{I}) \leq 14.0$ may arise from WHIM filaments and could be used to trace the overlaying ionized hydrogen component. The Ly $\alpha$ absorption from WHIM filaments is expected to be very broad due to thermal line broadening, resulting in large Doppler parameters of $b>40 \mathrm{~km} \mathrm{~s}^{-1}$. For pure thermal broadening, the $b$ value of the Ly $\alpha$ line is related to the temperature of the gas via $\log T \approx \log \left(60 b^{2}\right)$. In CIE, the ionized gas fraction $\left(f_{\mathrm{H}}=\mathrm{H}_{\text {total }} / \mathrm{H} \mathrm{I}\right)$ can be approximated via $\log f_{\mathrm{H}}(T) \approx-13.9+5.4 \log T-0.33(\log T)^{2}$ (Richter et al. 2004 [9]). Under the assumption that thermal broadening dominates the widths of the WHIM Ly $\alpha$ absorbers, one then can derive the total hydrogen mass of an individual absorber from its $b$ value and $\mathrm{H} \mathrm{I}$ column density. However, intergalactic Ly $\alpha$ absorbers with $b$ values $>40 \mathrm{~km} \mathrm{~s}^{-1}$ are generally difficult to detect, as they are broad and shallow. High resolution, high S/N FUV spectra of QSOs with smooth background continua are required to successfully search for broad Ly $\alpha$ absorption in the low-redshift WHIM. STIS (functional until 2004) was the only instrument available that delivered such data, but due to the instrumental limitations of space-based observatories, the number of QSO spectra eligible for searching for WHIM broad Ly $\alpha$ absorbers is very limited. The cosmological mass density of the broad Ly $\alpha$ absorbers at low $z, \Omega_{b}(\mathrm{BL})$, can be derived from the observed number statistics of broad Ly $\alpha$ absorbers in high-resolution STIS data. We can write:

$$
\Omega_{b}(\mathrm{BL})=\frac{\mu m_{\mathrm{H}} H_{0}}{\rho_{\mathrm{c}} c} \sum_{i j} f_{\mathrm{H}, i j} N(\mathrm{HI})_{i j} / \sum_{j} \Delta X_{j}
$$

with $\mu=1.3, m_{\mathrm{H}}=1.673 \times 10^{-27} \mathrm{~kg}, H_{0}=75 \mathrm{~km} \mathrm{~s}^{-1} \mathrm{Mpc}^{-1}$, and $\rho_{\mathrm{c}}=3 H_{0}{ }^{2} / 8 \pi G$. The index $i$ denotes an individual broad Ly $\alpha$ system along a line of sight $j$. Each measured absorption system 
$i$ is characterized by its neutral hydrogen column density, $N\left(\mathrm{H} \mathrm{I}_{i j}\right.$, and ionization fraction, $f_{\mathrm{H}, i j}$. Each line of sight $j$ has a characteristic redshift range, $\Delta X_{j}$, available for detecting broad Ly $\alpha$ absorption (see, e.g., Sembach et al. 2004 [7]).

We recently have measured the number density of broad Ly $\alpha$ absorbers at low $z$ in the directions of the quasars PG 1259+593, PG 1116+215, H 1821+643, and PG 0953+415, using highresolution STIS data (Richter et al. 2004 [9], Sembach et al. 2004 [7], Richter et al. 2005, in preparation) and have detected 26 candidate systems along a total redshift path of $\Delta z=0.939$. Fig. 1 shows an example of a broad Ly $\alpha$ absorber at $z=0.18047$ in the STIS spectrum of H 1821+643. The detected broad Ly $\alpha$ systems have H I column densities of $12.7 \leq \log N \leq 14.0$ and $b$ values ranging from 40 to $133 \mathrm{~km} \mathrm{~s}^{-1}$. Our measurements imply a number of broad Ly $\alpha$ systems per unit redshift of $d N_{\mathrm{BL}} / d z=28 \pm 5$ for $z<0.4$ and a cosmological mass density of $\Omega_{b}(\mathrm{BL}) \approx 0.0058 h_{75}^{-1}$, which is roughly twice the value obtained for the O VI absorbers. For this preliminary estimate we have omitted the contributions traced by the broadest Ly $\alpha$ absorbers with $b>100 \mathrm{~km} \mathrm{~s}^{-1}$. A large fraction of the baryons in the low redshift Universe may exist in those absorbers because the implied temperatures and ionization corrections are very large. However, absorbers with $b>100$ $\mathrm{km} \mathrm{s}^{-1}$ are difficult to study because of their weakness and large width. Note that for the above estimate of $\Omega_{b}(\mathrm{BL})$ we also have not considered any non-thermal line broadening mechanisms that may contribute to the observed large $b$ values. Such non-thermal processes include broadening by the Hubble flow, peculiar gas motions, and macroscopic turbulence. Future observational and theoretical investigations of $\Omega_{b}(\mathrm{BL})$ will need to address the influence of these processes for the determination of $T$ from the measured line widths. This will be crucial to test whether our current estimate of the baryon content of the broad Ly $\alpha$ absorbers is realistic.

\section{References}

[1] S.V. Penton, J.T. Stocke, \& J.M. Shull, The Local Ly $\propto$ Forest. IV. STIS G140M Spectra and Results on the Distribution and Baryon Content of HI Absorbers, ApJS 152, 29, 2004

[2] R. Davé, et al., Baryons in the Warm-Hot Intergalactic Medium, ApJ 552, 473, 2001

[3] M. Fukugita, Cosmic Matter Distribution: Cosmic Baryon Budget Revisited, astro-ph 0312517, 2003

[4] S. Burles \& D. Tytler, The Deuterium Abundance toward Q1937-1009, ApJ 499, 699, 1998

[5] D.N. Spergel, et al., First-Year Wilkinson Microwave Anisotropy Probe (WMAP) Observations: Determination of Cosmological Parameters, ApJS, 148, 175, 2003

[6] R. Cen \& J. Ostriker, Where Are the Baryons?, ApJ 514, 109, 1999

[7] K.R. Sembach, et al., Physical Properties and Baryonic Content of Low-Redshift Intergalactic Ly-alpha and O VI Absorption Systems: The PG1116+215 Sight Line, astro-ph 0407549, 2004

[8] B.D. Savage, et al., Discovery of a Hot, Multiphase NeVIII/OVI Absorber in the Low-redshift IGM Toward HE 0226-4110, ApJ, submitted, 2005

[9] P. Richter, et al., FUSE and STIS Observations of the Warm-hot Intergalactic Medium toward PG 1259+593, ApJS 153, 165, 2004

[10] R.S. Sutherland \& M.A. Dopita, Cooling Functions for Low-density Astrophysical Plasmas, ApJS 88, 253,1993 